\section{ADENOMA UTERI.}

Read in the Section of Obstetrics and Diseases of Women, at the Fortysecond Annual Meeting of the American Medical Association, at Washington, $U . C ., M a y, 189 t$.

BY HENRY C. COE, M.D., OF NEW YORK.

Introduction. - The writer formerly regarded true "malignant adenoma" of the corporeal endometrium as an extremely rare disease; late observation has convinced him that it is more common than he had supposed.

There has been very great confusion in the nomenclature of diseases of the endometrium. $\mathrm{Mu}$ cous polypi, endometritis fungosa, true adenoma and adeno-carcinoma, have all been described as varieties of adenoma. This is not only anatom. ically incorrect, but leads to serious error from a clinical standpoint. Even such an acute observer and clear writer as Winckel has introduced confusion into his description of the condition. Many diagnoses are made from a microscopical examination of curettings alone. In doubtful cases, no careful pathologist will positively commit himself on such insufficient evidence. The crucial test of malignancy is invasion of the submucous layer; this implies examination of sections of a growth made in situ, after removal of the entire uterus. The writer starts out with the proposition that there is only one variety of true adenoma, and that is essentially malignant clinical. So-called "adenomatous polypus," "benignant adenoma " and "endometritis fungosa," are not considered at all, since they are not true adenoma.

Brief Historical Review.-Matthews Duncan is credited with the first reported case of malignant adenoma, though the diagnosis was not supported by a subsequent autopsy. Breisky and Eppinger reported most thoroughly a bona fide case in 1877 , which has become classical. Schroeder wrote on the subject a few months later, describing two cases, and Veit confirmed his and Breisky's views regarding the ultimate transition of adenoma to adeno-carcinoma. Mąnn, Bertolet, 'Thomas and Goodell reported supposed cases, but Mann's description (case of Lusk's) is alone convincing. The most recent communications have been by Ruge, Veit and Fürst, the former having reported a case three years ago. The writer published a communication on the subject two years ago.

The writer has examined uteri removed by Wylie and Bache Emmet, which were undoubted specimens of pure adenoma uteri, that of Wylie's being an exquisite example of the diffuse form. $\mathrm{He}$ has himself extirpated two uteri in which the condition was circumscribed adenoma, and one in which there was a transition to adenoma-carcinoma. All five patients made a good recovery.

[Here follow brief histories and descriptions of the writer's specimens, which were to be presented to the Section.]
General deductions from the above cases:

Anatomy.-Unnecessary to describe the ordinary microscopical appearances of adenoma. The important point is that the malignant character of adenoma of the endometrium is shown by the fact that it is not confined to the mucosa, but invades

the submucous and muscular layers. Moreover, in some specimens (as in Breisky's), the transition to carcinoma has been clearly established.

Symptomatology. - There is no clearly defined set of symptoms peculiar to adenoma, but the writer does not agree with Ruge that they are never distinguishable from those of carcinoma corporis uteri. In the first place, the symptoms of adenoma are of longer standing than are those of primary carcinoma. There is less pain, the hæmorrhages are less frequent and profuse, and, so far as the writer's observation has extended, there is little or no foul-smelling, watery discharge. Glandular and perimetric involvement has been entirely absent in the four cases personally observed by the writer. The negative results of the examination of curettings is important, since there is seldom doubt in a well-marked case of carcinoma. Diagnosis. - This is sometimes extremely difficult, yet it is of more importance from a scientific than from a surgical standpoint. Given a patient who has passed the menopause, and who has for several months had slight atypical hæmorrhages with lancinating pelvic pains, even if a foul discharge, cachexia and other characteristic symptoms be wanting, we should make a thorough examination of the interior of the uterus by means of the finger and curette, after dilatation with tents. Finding a localized or diffuse papillary growth, we are justified in removing the uterus without delay, even if the microscope shows no positive evidence of malignant disease. Prognosis. - As the history of the few authentic cases of adenoma uteri has shown, the course of the disease is exceedingly slow and insidious. It is the least malignant of all the forms of malignant disease of the endometrium, but if left to itself, it inevitably assumes a more fatal form. We should not be misled by the long-continued good health of the patient, and the absence of profuse hæmorrhage, offensive discharges, and local pain and tenderness. The end, though long delayed, is certain.

Treatment.-Total extirpation of the uterus is the only treatment to be considered. Frequent curetting simply hastens the transition to the more malignant type of which adenoma is the initial stage. The patient is relieved for a time, but her symptoms soon reappear in an aggravated form. The use of the galvano-cautery, though more efficient, is no less objectionable. Remove the entire uterus. In cases of adenoma the operation is easy and uncomplicated, and there is a better prospect of a radical cure than in carcinoma, either of the cervix or corpus uteri. 
Summary._- "Benignant adenoma" is a mis. nomer. There is only one variety of true adenoma of the corpus uteri, and that is both clinically and anatomically malignant.

Adenoma uteri is not, at the outset, identical with adeno carcinoma, but is to be regarded as the initial stage of the latter. Its malignancy is shown by its early recurrence after removal with the sharp curette.

The symptoms of adenoma are less clearly defined than those of carcinoma, being usually slight irregular hæmorrhages, and pelvic pains extending over a long period, without cachexia and offensive discharges.

Palliative treatment is worse than useless. Total extirpation promises a radical cure if performed early, or during the pre-cancerous stage.

\section{LAPAROTOMY WITH REPORT OF CASES.}

Read in the Section of Obstatrics and Diseases of Women, at the Forly-second Annual Meeting of the American. Medical Association, held at Washington, D. C., May I8gI.

BY J. H. BRANHAM, M.D.,

OF BALTIMURE, MD.

The following paper is a report of the eleven laparotomies which I have done up to this time. They have all occurred during the past two years. The number is too small to be of much value from a statistical standpoint, but the difficulties met with in these cases are probably more keenly appreciated by a beginner than by an older operator, and I hope, by calling your attention to these, to make my report of value to those surgeons who are entering this field. could not be found. The bladder, which was adherent to the anterior part of the tumor, was drawn nearly up to the umbilicus, and was opened during the operation. It was carefully closed, and the patient made an uninterrupted recovery.

Case 2.-The right ovary was displaced into the inguinal region, was enlarged, softened, beginning to suppurate, and attached to the lower part of the small intestines by recent adhesions. In separating these, about one inch of the longitudinal diameter and more than half of the circumference of the gut was denuded of peritoneal covering and looked very dark, but this did not prevent a rapid convalescence.

Case 3.- Patient had suffered for thirteen years with symptoms of pelvic inflammation accompanied by dysmenorrhœa, fainting spells, etc. She had been confined to bed for months at a time, and was constantly in pain. The ovaries were atrophied and surrounded by many adhesions, and were enucleated with much difficulty. The wound healed by first intention, and although the patient has since continued to menstruate slightly her general health is much improved, and she suffers very little pelvic pain. Case 4.-This patient was a robust German woman, who, in addition to immęnse abscesses of both tubes and ovaries had a large abscess between the uterus and vagina in front and the rectum behind. In attempting to enucleate this several of the large pelvic veins were torn, and it was necessary to pack the pelvis with antiseptic gauze to control the hæmorrhage. The postmortem showed that the abscess behind the

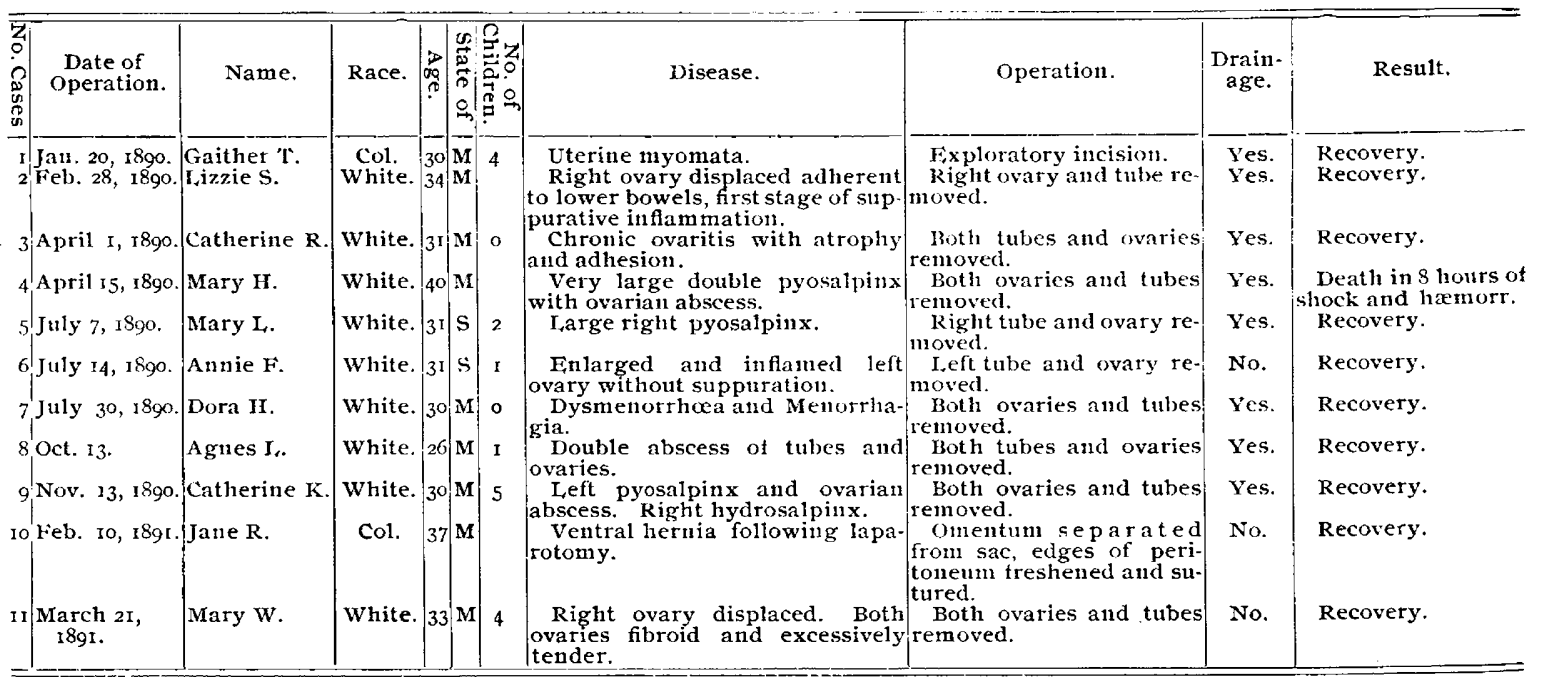

I will give, first, a tabulated history of the uterus could not have been enucleated without cases and then will call your attention to those fatal result, and simple drainage would have been points which seem to me to be of most interest. better.

Case 1 .- Operated with intention of removing Case 5.-Was a large pyosalpinx. Recovery ovaries and tubes, but the tumor in its growth rapid, and the patient is in excellent health at had surrounded them to such an extent that they the present time. 